\title{
Is granulovacuolar degeneration an essential pathological component of Alzheimer's disease? A review of the pathogenesis and histochemistry of old studies
}

\author{
Maher Kurdi ${ }^{1}$, Badrah Alghamdi² \\ ${ }^{1}$ Department of Pathology, Faculty of Medicine in Rabigh, King Abdulaziz University, Kingdom of Saudi Arabia, ${ }^{2}$ Department \\ of Physiology, Faculty of Medicine in Jeddah, King Abdulaziz University, Kingdom of Saudi Arabia
}

\begin{abstract}
Granulovacuolar degeneration (GVD) is a histopathological feature of Alzheimer's disease (AD) and some nonAlzheimer neurodegenerative diseases. It is also present in the brains of non-demented older adults. GVD is characterized by the presence of intracytoplasmic granule-containing vacuoles in most hippocampal neurons. It affects the neurons in sequential arrangement throughout the brain, which allows its characterization into different stages related to the severity of the disease. The mechanism of GVD formation is still poorly understood and its relationship with Tau structures remains unclear. Immunohistochemistry and ultrastructural examination suggest that GVD is mediated by cellular autophagic mechanisms. Other potential mechanisms related to GVD include protein accumulation caused by cellular defence mechanisms or impaired cellular functions. Several proteins are used as markers of GVD. Antibodies to cytoskeletal proteins and neurofilaments, both phosphorylated and non-phosphorylated forms, are used to stain GVD, the latter of which can be used to determine the nature of the cytoskeletal abnormalities in GVD formation. A link between GVD and microtubule-associated protein of tau was also reported but remains unclear. Previous studies reported neurons containing GVDs in the hippocampus of AD sections. Other neurodegenerative diseases also randomly showed GVDs in the brain. However, these quantitative studies have not demonstrated whether GVD is an essential component of $A D$ or non-AD dementias. In this review, we discuss our previous quantitative results of a retrospective study from 2016 and compare them with the results of older published studies to examine whether GVD is an essential feature of AD dementia or additional neurodegenerative features. We also revisit the pathogenesis and histochemistry profile of this common pathology.
\end{abstract}

Key words: granulovacuolar degeneration, Alzheimer's disease, neurodegenerative disease.

\section{Introduction}

Granulovacuolar degeneration (GVD) was first described in senile dementia by Simchowicz and Hirano in 1911 and 1986, respectively [13,17]. GVD was identified in the cornu-ammonis of the hippo- campus in $40 \%$ of cognitively normal patients aged 65-97 years [8]. Microscopically, GVD is defined as intraneuronal cytoplasmic vacuoles harbouring central granules [7] (Fig. 1), which are not associated with lipofuscin granules. Ultrastructurally, the large membrane-bound vacuoles are 3-5 $\mu \mathrm{m}$ in diameter

\section{Communicating author}

Maher Kurdi, MD, FRCPC, EFN, Department of Pathology, Faculty of Medicine in Rabigh, King Abdulaziz University,

Kingdom of Saudi Arabia, phone: +966 556655467, e-mail: Ahkurdi@kau.edu.sa 


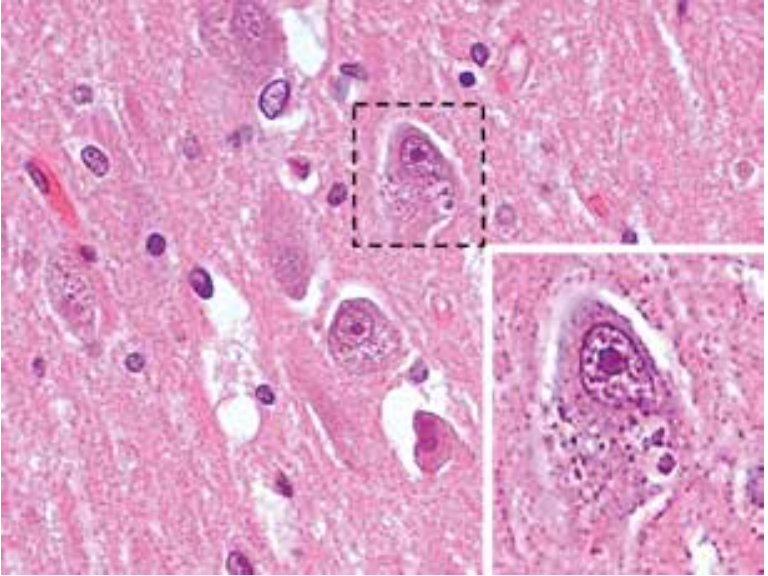

Fig. 1. Granulovacuolar degeneration (GVD) characterized by intraneuronal cytoplasmic vacuoles containing granules (H\&E 60x).

and contain argyrophilic granules 0.5-1.5 $\mu \mathrm{m}$ wide [31]. These granules can be distinguished from perisomatic granules (non-plaque dystrophic dendrites) because the latter contain glutamate receptor subunits and dense vesicles. The two-layered membrane structure of GVD suggests these lesions are of autophagic origin.

GVD is a histopathological feature of Alzheimer's disease (AD) and some non-Alzheimer neurodegenerative diseases such as progressive supranuclear palsy, pantothenate kinase-associated neurodegeneration, corticobasal degeneration and Pick's disease [44]. Moreover, there was a debate on whether GVD appeared with normal aging or it is associated with $A D$ [19]. It has been reported that GVD is an important component of $A D$ similar to the importance of senile plaque formation and neurofibrillary changes [19]. It has been found that the level of GVD in $A D$ brains is higher than that of aged-matched controls [19]. In this review, we focused on reviewing the updated studies of GVD to highlight its importance as a possible diagnostic parameter for AD.

\section{Pathogenesis of granulovacuolar degeneration}

The mechanism of GVD formation is poorly understood and its relationship with Tau structures remains unclear. Identification of the molecular basis of GVD is important to understand GVD formation and pathogenesis. Several studies have suggested that the sequestration of proteins into GVD might be caused by cellular defence mechanisms or impaired cellular functions [19]. These granulovacuolar granules contain epitopes from several constituent proteins in the autophagic ubiquitin proteasome system, suggesting a link between GVD and neurodegenerative diseases. Furthermore, GVD inclusions contain amphisomes, which are involved in autophagic and endocytic pathways, and which express similar epitopes. Only antibodies that recognize phospho-epitopes can detect proteins in granulovacuolar granules [4]. Several phosphorylated and non-phosphorylated proteins in GVD were previously identified. Therefore, GVD is thought to derive from cellular autophagic mechanisms related to some of these proteins. In autophagy, targeted proteins are polyubiquitylated, promoting binding to $p 62$, which induces autophagic protein microtubule-associated protein 1 light chain 3 (LC3) that helps assemble the autophagic membrane around the ubiquitylated cargo to form an autophagosome (Fig. 2). The autophagosome fuses with the lysosome directly or with multivesicular bodies to form amphisomes, which fuse with the lysosome to form an autolysosome, an organelle that degrades internal proteins and structural organelles [4]. Therefore, dysfunction of lysosomal fusion might be involved in GVD body formation. Failure of the lysosome to efficiently fuse with membrane-bound organelles might degrade captured proteins in the autophagic pathway and affect the intra-Tau concentrations leading to elevated p-tau levels [7].

Another theory by Castellani et al. suggests a novel link between oxidative stress damage and RNA metabolism, and GVD body formation in neurodegenerative diseases [11]. These intracellular bodies are referred to as stress granules that contain poly(A) mRNA, 40S ribosomal subunits, and p54/Rck protein. They occur after acute cellular stress when proteins are normally involved in mRNA processing, and activate mRNAs from disassembled polysomes [11]. This process occurs in stress granules, indicating granules are active molecular participants in cellular stress. Stress granules also contain RNA complexes suggesting they are involved in microRNA-induced translational pathways [11]. Furthermore, oxidative stress in neurons induces extracellular regulated kinase (ERK) activation, a protein present in GVD that induces $\mathrm{S} 6$ phosphorylation via S6 kinase stimulation [11], the absence of which is associated with low cell survival. Funk et al. reported that the endocytic mechanism involved in the formation of GVD is 


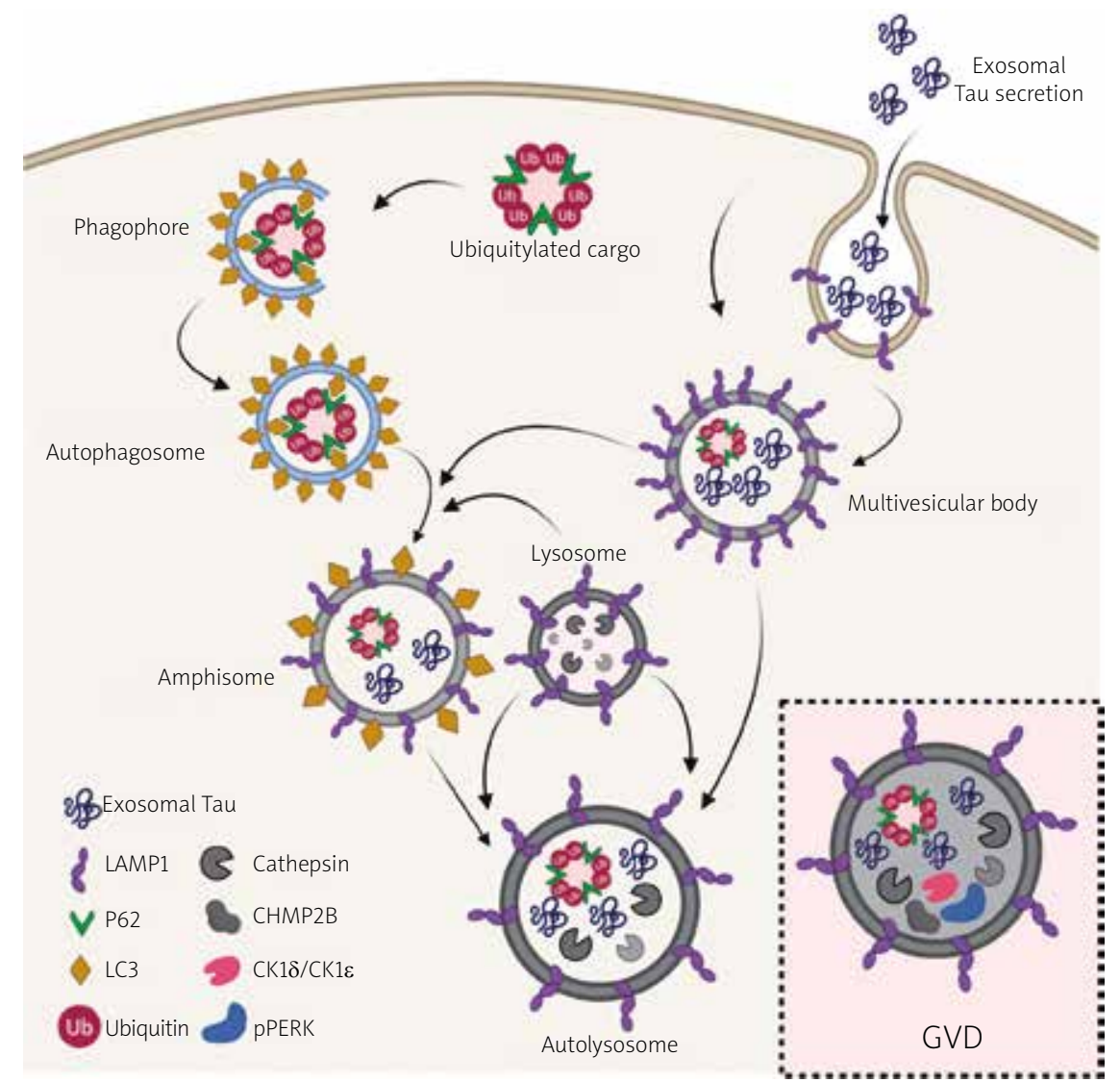

Fig. 2. Intracellular vesicular trafficking pathways (cellular autophagic mechanism) involved in granulovacuolar degeneration (GVD) formation.

associated with strong immunoreactivity of charged multivesicular body protein 2B (CHMP2B), a component of endosomal sorting complex required for transport (ESCRT-III) in the GVD body core $[7,41]$. This suggests that a failure of multivesicular bodies to fuse with the lysosome may promote the accumulation of autophagic intermediates and increased size of multivesicular bodies [7]. Frontotemporal dementia, an autosomal dominant disease linked to chromosome 3 (FTD-3), is rarely caused by the CHMP2B gene [15]. Casein kinase 1 (CK1) isoforms and CHMP2B appear to be useful markers for GVD.

Recently, Murata-Shinozaki et al. found a correlation between GVD bodies and a set of common molecules, suggesting GVD originates from the postsynaptic spine, especially in terms of the Wnt signalling pathway [28]. The role of the postsynaptic spine in GVD body formation was demonstrated by immunostaining and immunofluorescence in all cases of AD. GVDs were pathologically reactive for dishevelled family proteins including phospho- $\beta$-catenin, ribo- sylation factor 6 (rapsyn), adenomatous polyposis coli (APC) and PCNA-interacting protein (PIP2) [28]. These findings indicate that GVD bodies originate from Wnt signalling pathway dysfunction [28]. $\beta$-catenin levels are highly regulated in the cytosol and it plays a crucial role in regulating the canonical Wnt-pathway. The levels of punctate cytoplasmic inclusions for phosphorylated $\beta$-catenin were significantly higher in AD patients compared with control samples suggesting GVD inclusion formation is mediated by dysfunctional proteasome pathways [9].

\section{Pathological staging of granulovacuolar degeneration}

Granulovacuolar degeneration pathology affects neurons in the brain and expands throughout the brain via a specific sequence of brain regions, which is used to characterize it into five stages. This staging system allows the scoring of the severity of GVD and independent neuronal death. Thal et al. [38] 
described the staging system of GVD distribution as follows (Fig. 3):

- Stage 1: GVD occurs only in the CA1, CA2, and subiculum regions;

- Stage 2: GVD is present in the CA1, CA2, subiculum, entorhinal cortex, and/or CA4 and CA3 regions, as well as the basal nucleus of Meynert;

- Stage 3: Stage 2 regions plus the temporal neocortex;

- Stage 4: Stage 3 regions plus GVD in the subnuclei of amygdala, and/or the hypothalamic nuclei, cingulate gyrus, and insular cortex;

- Stage 5: GVD is present in all previous stages areas plus GVD in the dorsal raphe nuclei, pedunculopontine tegmental nucleus, cingulate gyrus, and occasionally in the superficial and medial nuclei of the thalamus, frontal and parietal neocortex.

\section{Link between tau filaments and granulovacuolar degeneration}

The relationship between GVD and neurofibrillary tangle (NFT) in hippocampal neurons in $A D$ is unknown [3]. The fusion of lysosomes or endosomes with autophagosomes is mediated by intact microtubules. Therefore, any disruption in the microtubule network might facilitate tau and GVD formation. It was reported that the number of neurons containing GVDs correlated directly with those containing NFTs and was inversely correlated with the number of pyramidal cells. However, there was no direct link between GVD and NFT formation, although both are expressed with increasing age [40]. GVD and NFT are immunoreactive for ubiquitin and phosphorylated tau. Nevertheless, not all tau-directed antibod-
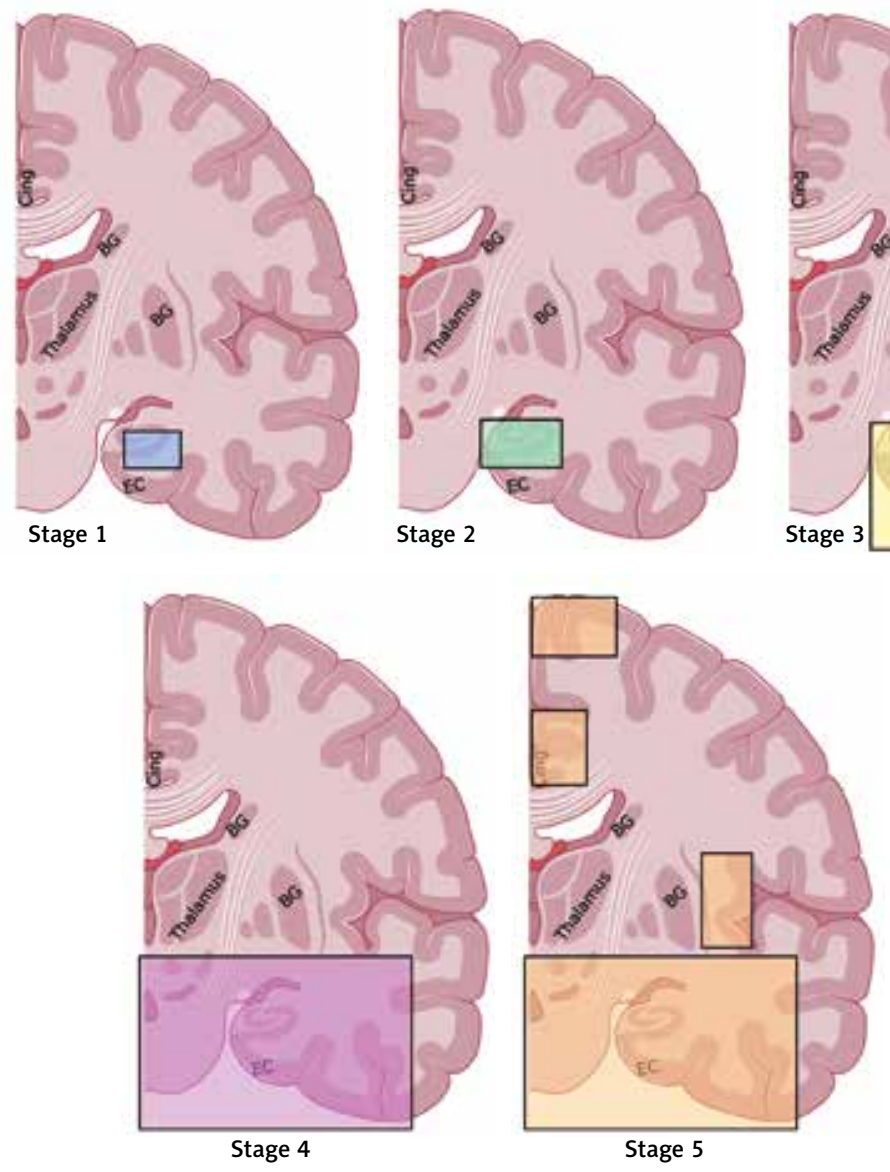

Fig. 3. The staging system of the anatomical distribution of granulovacuolar degeneration (GVD) throughout the brain. Stage 1, GVD in the CA1, CA2, and subiculum regions. Stage 2, stage 1 plus GVD in the entorhinal cortex, CA4, CA3, and basal nucleus of Meynert. Stage 3, stage 2 plus GVD in the temporal cortex. Stage 4, stage 3 plus GVD in the amygdala and hypothalamus. Stage 5, stage 4 plus the cingulate and insular cortex, dorsal raphe nuclei, pedunculopontine tegmental nucleus, and occasionally in the superficial and medial nuclei of the thalamus, and frontal and parietal neocortex. 
ies that immunolabel NFTs also label GVD granules. Indeed, the presence of the complete tau molecule in a GVD complex and NFT suggests the GVD granule may represent a site of tau, perhaps related to pair helical filament (PHF) formation in the hippocampus. In addition, glycogen synthase kinase-3 (GSK-3) was reported to have an important role in the phosphorylation of tau, cell growth, development, and fate regulation. Leroy et al. found that GVD was strongly reactive to GSK-3 and the active form of each GSK [23]. Therefore, GSK-3 is present in the cytoplasm of neurons containing NFT. Because GVD is considered a unique type of autophagosome, GSK-3-positive GVD represents the segregation of active GSK-3 in this cell compartment. Gosphal et al. established a link between the fibrillar (tau filaments) and GV pathologies of AD based on molecular analyses; both lesions accumulated CK-1 protein [11]. The association between CK-1 and tau filament appears to be physical because it is maintained through stringent purification methods. However, CK-1 immunolabelling was not mediated by phosphoepitopes and the GVD lesion was accompanied by essential increases in the amounts of these protein kinases. The location of these identified CK-1 isoforms suggests they might play a role in generating phosphoepitopes in GVD and neurofibrillary structures. Although the precise molecular mechanism of CK-1 isoform trafficking is unknown, the intracellular distribution of CK-1 isoforms suggests that they exert their effects at the level of the cytoskeleton. Therefore, elevated levels of CK-1 might hyperphosphorylate substrates at the cytoskeleton membrane.

These data suggest that GVD formation occurs when phosphorylated tau begins to aggregate into early-stage neurofibrillary tangles [35]. Therefore, GVD is the main site of tau phosphorylation. Because abnormal glycosylation may assist the hyperphosphorylation of tau, we consider p-tau in
GVD is hypersialylated [24]. Furthermore, anti-sialic acid antibody specifically identified NFTs and GVDs demonstrating immunostaining for sialylation represents a useful diagnostic tool to screen for GVD [29]. However, the relationship between GVD and the microtubule-associated phosphoprotein of tau remains elusive [13].

\section{Is granulovacuolar degeneration an essential pathological component in the microscopic diagnosis of Alzheimer's disease?}

The amount of GVD increases with disease severity and memory decline [7]. This is not merely specific to $A D$ or the hippocampus, although its association with both is characteristic and well established [3]. Two primary cardinal microscopic lesions associated with $A D$ are the NFT and the senile plaque (Table I). The absence of one of these major features in demented patients with a clinical suspicion of $A D$ may rule out the diagnosis. Other neuropathological lesions such as GVD and Hirano body are considered additional features for the diagnosis of AD. Many retrospective studies (quantitative vs. qualitative) have attempted to define the prevalence and distribution of GVDs in normally-aged and neurodegenerative disease brains to investigate whether GVD is a major or minor pathological component in the diagnosis of neurodegenerative diseases; mainly AD or/and Lewy body disease (LBD). The topographic predilections of GVDs in the hippocampal cortex of aged or demented brains are not clear. GVD incidence is highest in the hippocampal region suggesting the metabolic properties of hippocampal neurons foster GVD particle formation. The hippocampus has a major role in cognitive function; therefore, its pathological impairment contributes significantly to dementia [19]. Rose et al. analysed GVDs in defined fields of the hippo-

Table I. Pathological criteria for the diagnosis of Alzheimer disease

\begin{tabular}{|ccc|}
\hline & Pathological criteria & Braak stage \\
\hline \multirow{2}{*}{ Major criteria } & Intracellular NFT & Thal and CERAD stage \\
\cline { 2 - 3 } & Extracellular amyloid plaques & Thal GVD stage \\
\hline Minor criteria & Granulovacuolar degeneration \\
\cline { 2 - 3 } & Hirano body \\
\cline { 2 - 3 } & Amyloid angiopathy & \\
\cline { 2 - 3 } & Status spongiosis & \\
\hline
\end{tabular}

NFT - neurofibrillary tangles, CERAD - Consortium to Establish a Registry for Alzheimer's Disease 
campus [27]. Within the hippocampus proper, GVD pathology was mainly present in the CA1 and CA2 regions. In cases where several hippocampal neurons were affected, GVDs were only identified throughout the entire pyramidal layer, usually in both hemispheres. Tomlinson and Kitchener et al. measured the degree of GVD in 219 brains from cognitively-normal and demented individuals aged 14-98 years [40]. The distribution of GVD in the hippocampal cortex of 30 non-demented elderly people and 25 patients with senile dementia was similar. Only two cases below the age of 50 years had neurons affected with GVD. The main anatomical location was similar to that reported by Xu et al., although they found that 54 of 75 non-demented cases aged $>60$ years harboured GVD in the CA1 and CA2 regions, and only one case of 21 patients aged < 60 years had GVD [43]. They suggested that the difference between the numbers of neurons affected with GVD in $A D$ and non-AD dementia was statistically significant. Woodard et al. also reported that the ventrolateral quadrant of the hippocampus was more vulnerable to GVD compared with the rest of the hippocampus, especially the parahippocampus cortex [42]. However, they found that $9 \%$ of neurons with GVDs in the hippocampus were specific to $A D$ patients. Therefore, the Tomlinson analysis did not confirm the absolute distinction of the $9 \%$ level found by Woodard, although they confirmed the number of hippocampal neurons affect- ed with GVD distinguished $A D$ or senile dementia from non-demented controls. In a cohort of 37 cases (12 with normal cognitive brains, 12 with mild impairment, and 13 with $A D$ ), the degree of CK1 1 -labelling of GVDs in CA1 correlated significantly with the neuropathological diagnosis but not with the defined classifications [19].

Thal et al. assessed the distribution of GVD in the brains of 766 autopsy cases and found TDP43-pSer409/410-immunostained GVD in 96.3\% of $A D$ cases, $46.2 \%$ of preclinical $A D$ cases, and $28.6 \%$ of non-AD control cases [39]. This indicated that non-AD decedents with absent amyloid plaques, but with early NFT and GVD, might have preclinical $A D$ that precedes plaque pathology. Ball et al. reported that the CA1 and subiculum zones of the hippocampus were the most susceptible to GVD development [2]. Xu et al. [43] and Is et al. [14] performed studies on human and rat experimental model brains, respectively. A qualitative analysis demonstrated that neurons affected with GVD in cases of $A D$ were present in the entire examined areas outside the hippocampus and, in contrast to the results in $A D$, there were no neurons affected with GVD in cases of mild cognitive impairment. According to Ball et al., GVDs were predominantly present in the order CA1, subiculum, and CA2 [1]. AD cases had higher values than controls. Interestingly, when the magnitude of involvement of GVD and

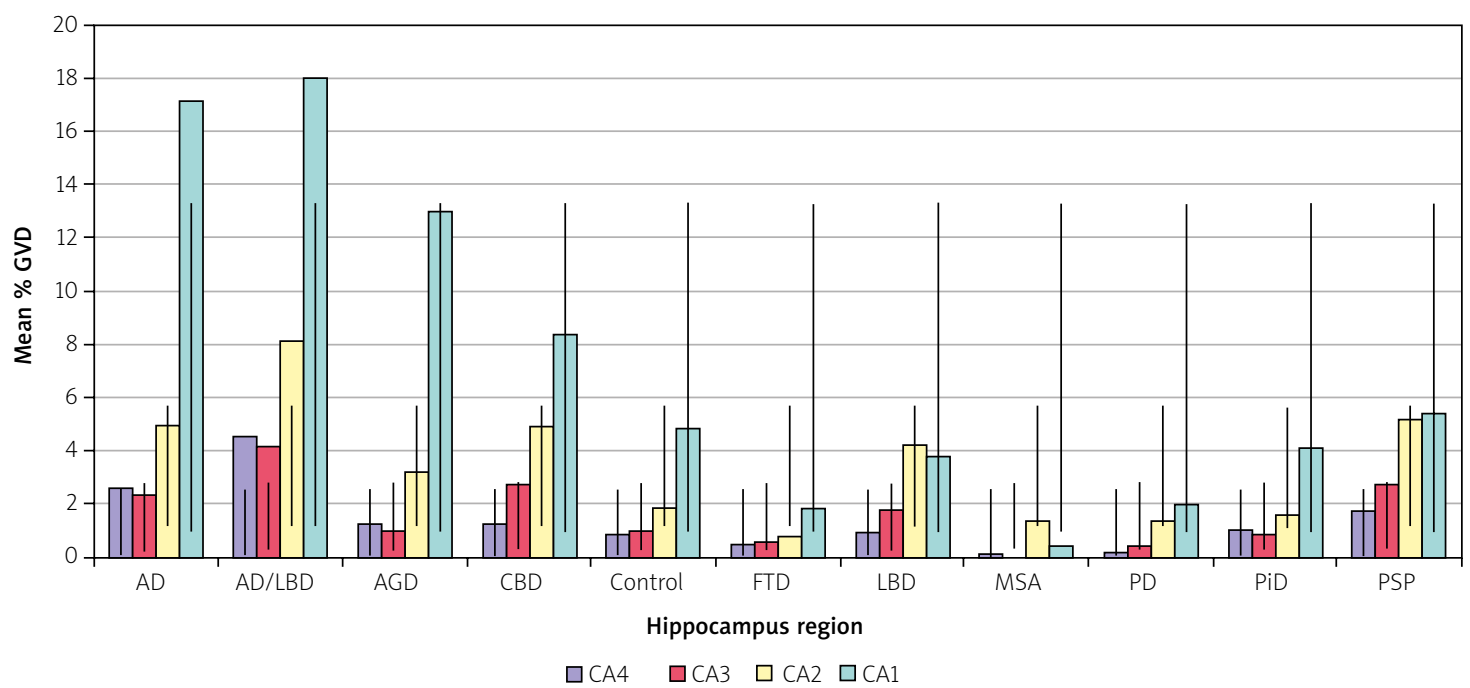

Fig. 4. Statistical distribution of granulovacuolar degeneration (GVD) in the hippocampus. The graph shows the accumulative mean percentages of GVD prevalence in each hippocampus sector in cases of neurodegenerative disease [21]. AD - Alzheimer's disease, LBD - Lewy body disease, AGD - argyrophilic grain disease, CBD - corticobasal degeneration, FTD - frontotemporal degeneration, MSA - multiple system atrophy, PD - Parkinsonism disease, PiD - Pick's disease, PSP - progressive supranuclear palsy. 
NFTs between AD cases and controls in the six zones was compared, the difference for GVD and NFTs was largest in CA2, followed by the subiculum and CA1 [1]. Keage et al. reported the high prevalence of GVDs in the hippocampus compared with the entorhinal cortex in cases with or without dementia [18]. Our recent 2016 study investigated 112 autopsied neurodegenerative cases and 70 control cases with age-related changes (aged 43-95 years) between 1993 and 2005. The quantitation mainly focused on the mean percentage of affected neurons with GVD and whether these changes were age- or disease-related [21]. We found that neurons with GVD were predominantly present in the CA1 and CA2 regions. The GVD count in $A D$ was significantly increased in the CA1 and $C A 2$ regions compared with other neurodegenerative diseases. There was a significant increase in GVDs in the CA1 region of $A D / L B D$ cases but there was no significant change in LBD cases. Therefore, the frequency of GVDs in $A D$ is related to the disease process and explains the increase of AD/LBD in AD cases (Fig. 4). Other diseases (argyrophilic grain disease [AGD], corticobasal degeneration [CBD], frontotemporal degeneration [FTD], multiple system atrophy [MSA], Parkinsonism disease [PD], Pick's disease [PiD], and progressive supranuclear palsy [PSP]) had various percentages of GVD present in all hippocampal sectors. However, these cases did not differ significantly from demented cases suggesting that GVD was associated with aging. Taken together, we concluded that GVD might be a consistent major finding in cases with Alzheimer-type pathology. Although the prevalence of GVD is low outside the hippocampus, GVD follows the same sequential pattern of NFT distribution. This suggests tau structures may increase GVD formation, particularly in hippocampal neurons. In conclusion, neurons in the CA1 and CA2 regions of the hippocampus are most susceptible to the development of GVDs whereas neurons in the end plate of the hippocampus are less frequently affected by GVD [1]. Low amounts of GVD were observed in the neocortex, hypothalamus, amygdala, and paramedian nuclei of the midbrain [36]. Although GVD in pyramidal cells of the hippocampus is always associated with senile dementia, this change was also observed with increasing severity as age advances. Diseases such as PSP, PiD, PD, Parkinson's disease of Guam, and Down syndrome showed variable amounts of GVDs in the hippocampus and other regions [3,13], which correlated with the severity of dementia.

\section{Histochemical profile of granulovacuolar degeneration}

Simchowicz characterized GVDs using several immunohistochemical or special staining methods. Although GVD granules are easily detected using non-specific histological stains such haematoxylin and eosin (H\&E), they also exhibit reactivity to silver impregnation stains (Bielschowsky stain) [21] (Fig. 5A, B). The Papanicolaou preparation highlighted few neurons with GVD [37]. Furthermore, GVDs

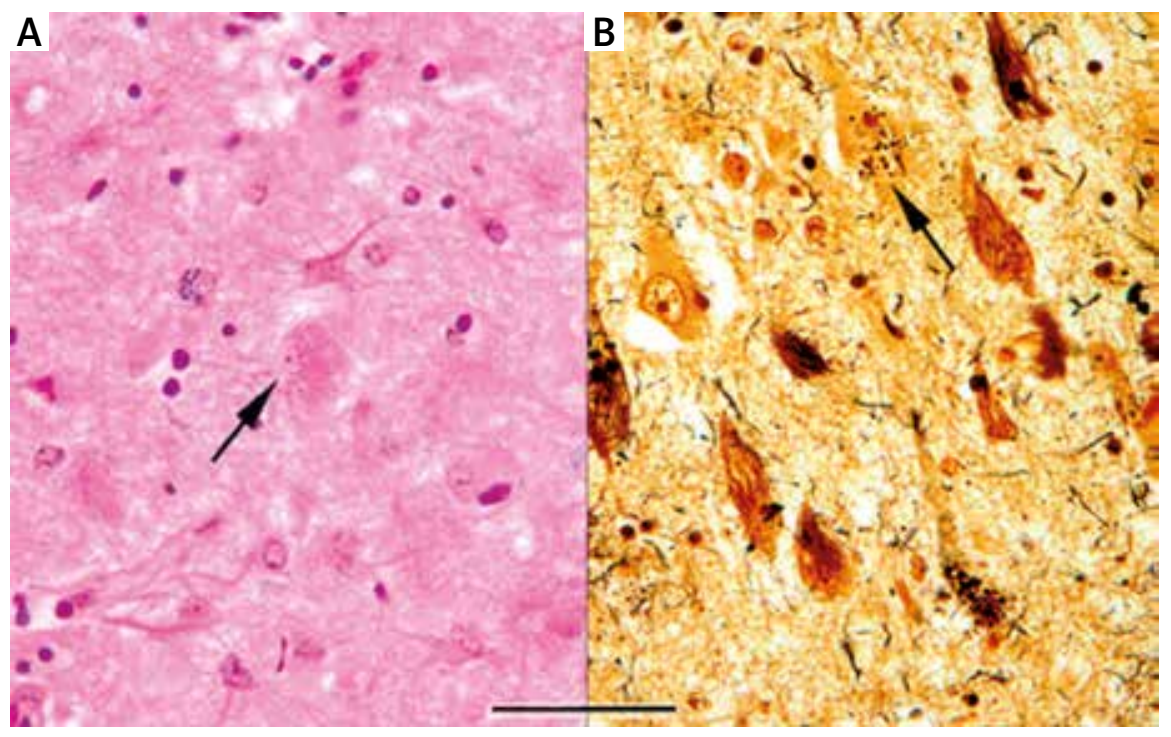

Fig. 5. Granulovacuolar degeneration (GVD). A) H\&E 60x; B) Bielschowsky 60x. 
were not stained with Sudan dye, Congo red, or Periodic acid-Schiff (PAS) staining methods.

Antibodies to cytoskeletal proteins were the first reported to stain GVD inclusions; this included antibodies to phosphorylated and non-phosphorylated neurofilaments. Phosphorylated tau, ubiquitin, tropomyosin, vimentin, and, interestingly mitotic proteins have been also found in GVD [27]. Antibodies to an alkaline phosphatase-associated antigen were shown to label GVD inclusions [10]. Other studies reported the diffuse immunoreactivity of GVDs when using phosphorylation-dependent protein antibodies such as tau [23], TDP-43 [16], ubiquitin [25], GSK [23], and CHMP2B [9]. However, antibodies to Tau-2 recognized a conformation-dependent epitope around Ala106-Lys119 of human tau [22] but failed to react with GVD granules. This was also reported in cases that did not stain GVDs [12]. However, the antibodies BR133 and BR134 labelled GVD granules. Neurons in the CA1 and subiculum of AD samples demonstrated strong CHMP2B-immunoreactivity that was morphologically similar to GVD staining. Neurons in several neurodegenerative disorders also showed CHMP2B-positive GVD bodies [44]. Nakamori et al. reported that the CDK5 immunomarker was a novel label for GVD bodies [30]. Dickson et al. also showed that GVD was immunoreactive to phosphorylated-dependent neurofilament protein [6]. A study by Siedlak et al. found that transmembrane Protein 230 (TMEM230) was present in GVDs and dystrophic neuritis. These findings suggest that TMEM230 is a component of GVD and might be involved in the pathogenesis of AD [34]. Newly-identified protein antibodies related to Wnt signalling pathways such as dvl, phospho--catenin, rapsyn, APC, and PIP2 strongly stained GVD bodies when used for immunohistochemistry and immunofluorescence [28]. Nagamine et al. identified an anti-sialic acid antibody as a specific immunomarker for GVD related to the hypersialylation of p-tau [29]. Several Tau protein kinases including GSK-3 and cyclin-dependent kinase 5 were also detected in GVD granules [32]. Clustering of deleterious kinases in GVD inclusions may represent a protective neurological mechanism. Only two studies have detected amyloid precursor protein as a major component of senile plaques and as an essential component in the pathogenesis of AD. An antibody against a phosphorylated form of the A -peptide showed diffuse staining for GVD granules [20]. Immunohistochemical analysis of cAMP-response element-binding protein (CREB) showed strong immunoreactivity and the number of neurons with GVD inclusions labelled by phosphorylated CREB was significantly higher in AD cases [33]. Neurons with Pin1-immunoreactive granules were also found in GVD bodies, but only in hippocampal neurons [5]. Several cellular stress-related proteins including p38 mitogen-activated protein kinase, c-Jun, and small ribosomal subunit protein 6 , were also detected in neurons with GVDs [45]. Lund et al. assessed microtubule affinity and the regulation of kinases (MARK2 and MARK4) in early tau pathology and GDV bodies [26]. Immunohistochemical analyses using isoform-selective antibodies demonstrated that phosphorylated MARK4 colocalized with p-tau Ser262 in GVDs that had accumulated in AD. The expression of phosphorylated forms of MARK4 in GVDs of AD samples was highly correlated with the phosphorylation of tau at Ser262. This may act as a cellular defence mechanism to remove activated MARK from the cytosol, thereby reducing the phosphorylating effect on tau Ser262 [26]. GVD markers, including lipid raft-associated proteins caspase 3 , LRRK2, annexin 2, and flotillin-1 were also detected on the rim of GVDs [30].

\section{Conclusions}

Pyramidal neurons in the CA1, CA2, and subiculum regions of the hippocampus are more likely to contain GVDs than neurons in the end plate of the hippocampus (CA3 and CA4 regions). Low amounts of GVD were observed in other brain regions. GVD in pyramidal cells of the hippocampus is frequently associated with AD, although it has been observed in non-demented elderly people. Other non-AD neurodegenerative diseases showed variable amounts of GVDs in the hippocampus and other regions, which correlated with the severity of dementia. On the basis of the previously reported quantitative studies, we conclude that GVD is an important criterion to diagnose early Alzheimer dementia. Further statistical studies should be conducted for AD cases with GVD based on the clinical diagnosis of AD. And a deeper understanding of the pathogenesis of GVD and its relation to tau pathology and $A D$ progression is needed to be investigated in future researches.

\section{Disclosure}

The authors report no conflict of interest. 


\section{References}

1. Ball MJ. Topographic distribution of neurofibrillary tangles and granulovacuolar degeneration in hippocampal cortex of aging and demented patients. A quantitative study. Acta Neuropath 1978; 42: 73-80.

2. Ball MJ, Lo P. Granulovacuolar degeneration in the ageing brain and in dementia. J Neuropathol Exp Neurol 1977; 36: 474-487.

3. Bondareff W, Wischik CM, Novak M, Rotht M. Sequestration of Tau by granulovacuolar degeneration in Alzheimer's disease. Am J Pathol 1991; 139: 641-647.

4. Caltagarone J, Hamilton RL, Murdoch G, Jing Z, DeFranco DB, Bowser R. Paxillin and hydrogen peroxide-inducible clone 5 expression and distribution in control and Alzheimer disease hip pocampi. J Neuropathol Exp Neurol 2010; 69: 356-371.

5. Dakson A, Yokota O, Esiri M, Bigio EH, Horan M, Pendleton N, Richardson A, Neary D, Snowden JS, Robinson A, Davidson YS, Mann DM. Granular expression of prolyl-peptidyl isomerase PIN1 is a constant and specific feature of Alzheimer's disease pathology and is independent of tau, A and TDP-43 pathology. Acta Neuropathol 2011; 121: 635-649.

6. Dickson DW, Ksiezak-Reding H, Davies R, Yen SH. A monoclonal antibody that recognizes a phosphorylated epitope in Alzheimer neurofibrillary tangles, neurofilaments and tau proteins immunostains granulovacuolar degeneration. Acta Neuropathol 1987; 73: 254-258.

7. Funk K, Mrak R, Kuret J. Granulovacuolar degeneration bodies of Alzheimer's disease resemble late stage autophagic organelles. Neuropathol Appl Neurobiol 2011; 37: 295-306.

8. Gellerstedt N. Zur Kenntnis der Hirnveränderungen bei der normalen Altersinvolution. Inauguraldissertation. Upsala Läkareförenings Förhandlingar 1933; 38: 5-6.

9. Ghanevati M, Miller CA. Phospho-beta-catenin accumulation in Alzheimer's disease and in aggresomes attributable to proteasome dysfunction. J Mol Neurosci 2005; 25: 79-94.

10. Gheuens J, Cras P, Perry G, Boons J, Ceuterick-de Groote C, Lübke U, Mercken M, Tabaton M, Gambetti PL, Vandermeeren M, Mulvihill P, Siedlak S, Heuverswijn HV, Martin J. Demonstration of a novel neurofilament associated antigen with the neurofibrillary pathology of Alzheimer and related diseases. Brain Res 1991; 558: 43-52.

11. Ghoshal N, Smiley JF, DeMaggio AJ, Hoekstra MF, Cochran EJ, Binder LI, Kuret J. A new molecular link between the fibrillar and granulovacuolar lesions of Alzheimer's disease. Am J Pathol 1999; 155: 1163-1172.

12. Grundke-Iqbal I, Iqbal K, Tung YC, Quinlan M, Wisniewski HM, Binder LI. Abnormal phosphorylation of the microtubule-associated protein tau (tau) in Alzheimer cytoskeletal pathology. Proc Natl Acad Sci USA 1986; 83: 4913-4917.

13. Hirano A, Dembitzer HM, Kurland LT, Zimmerman HM. The fine structure of some intraganglionic alterations. Neurofibrillary tangles, granulovacuolar bodies and "rod-like" structures as seen in Guam amyotrophic lateral sclerosis and Parkinsonism dementia complex. J Neuropathol Exp Neurol 1968; 27: 167-182.

14. Is M, Comunoglu NU, Comunoglu C, Ereb B, Ekici ID, Ozkan F. Age-related changes in the rat hippocampus. J Clin Neurosci 2008; 15: 568-574.
15. Isaacs AM, Johannsen P, Holm I, Nielsen JE. Frontotemporal dementia caused by CHMP2B mutations. Curr Alzheimer Res 2011; 8: 246-251.

16. Kadokura A, Yamazaki T, Kakuda S, Makioka K, Lemere CA, Fujita Y, Takatama M, Okamoto K. Phosphorylation-dependent TDP-43 antibody detects intraneuronal dot-like structures showing morphological characters of granulovacuolar degeneration. Neurosci Lett 2009; 463: 87-92.

17. Kahn J, Anderton BH, Probst A, Ulrich J, Esiri MM. Immunohistological study of granulovacuolar degeneration using monoclonal antibodies to neurofilament. J Neurol Neurosurg Psychiatry 1985; 48: 924-926.

18. Keage HA, Ince PG, Matthews FE, Wharton SB, McKeith IG, Brayne C; MRC CFAS and CC75C. Impact of less common and "disregarded" neurodegenerative pathologies on dementia burden in a population-based cohort. J Alzheimers Dis 2012; 28: 485-493.

19. Köhler C. Granulovacuolar degeneration: a neurodegenerative change that accompanies tau pathology. Acta Neuropathol 2016; 132: 339-359.

20. Kumar S, Wirths O, Stüber K, Wunderlich P, Koch P, Theil S, Rezaei-Ghaleh N, Zweckstetter M, Bayer TA, Brüstle O, Thal DR, Walter J. Phosphorylation of the amyloid -peptide at Ser26 stabilizes oligomeric assembly and increases neurotoxicity. Acta Neuropathol 2016; 131: 525-537.

21. Kurdi M, Chin E, Ang LC. Granulovacuolar degeneration in hippocampus of neurodegenerative diseases: quantitative study. J Neurodegener Dis 2016; 2016: 6163186.

22. Lang E, Otvos L Jr. A serine $\rightarrow$ proline change in the Alzheimer's disease-associated epitope Tau 2 results in altered secondary structure, but phosphorylation overcomes the conformational gap. Biochem Biophys Res Commun 1992; 188: 162-169.

23. Leroy K, Boutajangout A, Authelet M, Woodgett J, Anderton B, Brion J. The active form of glycogen synthase kinase-3 is associated with granulovacuolar degeneration in neurons in Alzheimer's disease. Acta Neuropathol 2002; 103: 91-99.

24. Liu F, Zaidi T, Iqbal K, Grundke-lqbal I, Merkle RK, Gong CX. Role of glycosylation in hyperphosphorylation of tau in Alzheimer's disease. FEBS Lett 2002; 512: 101-106.

25. Love S, Saitoh T, Quijada S, Cole GM, Terry RD. Alz-50, ubiquitin and tau immunoreactivity of neurofibrillary tangles, Pick bodies and Lewy bodies. J Neuropathol Exp Neurol 1988; 47: 393-405.

26. Lund H, Gustafsson E, Svensson A, Nilsson M, Berg M, Sunnemark D, von Euler G. MARK4 and MARK3 associate with early tau phosphorylation in Alzheimer's disease granulovacuolar degeneration bodies. Acta Neuropathol Commun 2014; 2: 22.

27. Man DM. Granulovacuolar degeneration in pyramidal cells of the hippocampus. Acta Neuropath 1978; 42: 149-151.

28. Murata-Shinozaki Y, Takahashi T, Matsubara T, Maruyama H, Izumi Y, Matsumoto $M$. The origins of rimmed vacuoles and granulovacuolar degeneration bodies are associated with the Wnt signaling pathway. Neurosci Lett 2017; 638: 55-59.

29. Nagamine S, Yamazaki T, Makioka K, Fujita Y, Ikeda M, Takatama M, Okamoto K, Yokoo H, Ikeda Y. Hypersialylation is a common feature of neurofibrillary tangles and granulovacuolar degeneration in Alzheimer's disease and tauopathy brains. Neuropathology J 2016; 36: 333-345. 
30. Nakamori M, Takahashi T, Nishikawa T, Yamazaki Y, Kurashige T, Maruyama H, Arihiro K, Matsumoto M. Molecular Markers for granulovacuolar degeneration are present in rimmed vacuoles. PLoS One 2013; 8: e80995.

31. Okamoto K, Hirai S, lizuka T, Yanagisawa T, Watanabe M. Reexamination of granulovacuolar degeneration. Acta Neuropathol 1991; 82: 340-345.

32. Pei JJ, Braak E, Braak H, Grundke-Iqbal I, Iqbal K, Winblad B, Cowburn RF. Distribution of active glycogen synthase kinase 3 beta (GSK-3beta) in brains staged for Alzheimer disease neurofibrillary changes. J Neuropathol Exp Neurol 1999; 58: 1010-1019.

33. Satoh J, Tabunoki H, Arima K. Molecular network analysis sug gests aberrant CREB-mediated gene regulation in the Alzheimer disease hippocampus. Dis Mark 2009; 27: 239-252.

34. Siedlak SL, Jiang Y, Huntley ML, Wang L, Gao J, Xie F, Liu J, Su B, Perry G, Wang X. TMEM230 accumulation in granulovacuolar degeneration bodies and dystrophic neuritis of Alzheimer's disease. J Alzheimer Dis 2017; 58: 1027-1033.

35. Stadelmann C, Deckwerth TL, Srinivasan A, Bancher C, Brück W, Jellinger K, Lassmann $\mathrm{H}$. Activation of caspase-3 in single neurons and autophagic granules of granulovacuolar degeneration in Alzheimer's disease: evidence for apoptotic cell death. Am J Pathol 1999; 155: 1459-1466.

36. Steele JC, Richardson JC, Olszewski J. Progressive Supranuclear Palsy. A heterogeneous degeneration involving the brain stem, basal ganglia and cerebellum with vertical gaze and pseudobulbar palsy, nuchal dystonia and dementia. Arch Neurol 1964; 10: 333-359.

37. Takei H, Kosarac O, Powell SZ. Cytomorphologic manifestations of Alzheimer's disease using brain squash smears: an autopsy study with histology-cytology correlation. Diagn Cytopathol 2009; 37: 654-660.

38. Thal DR, Del Tredici K, Ludolph AC, Hoozemans JJ, Rozemuller AJ, Braak H, Knippschild U. Stages of granulovacuolar degeneration: their relation to Alzheimer's disease and chronic stress response. Acta Neuropathol 2011; 122: 577-589.

39. Thal DR, von Arnim C, Griffin WS, Yamaguchi H, Mrak RE, Attems J, Upadhaya AR. Pathology of clinical and preclinical Alzheimer's disease. Eur Arch Psychiatry Clin Neurosci 2013; 263: S137-S145.

40. Tomlinson BE, Kitchener D. Granulovacuolar degeneration of hippocampal pyramidal cells. J Pathol 1972; 106: 165-185.

41. Wiersma VI, Scheper W. Granulovacuolar degeneration bodies: red alert for neurons with MAPT/tau pathology. Autophagy 2020; 16: 173-175.

42. Woodard JS. Clinicopathologic significance of granulovacuolar degeneration in Alzheimer's disease. J Neuropathol Exp Neurol 1962; 21: 85-91.

43. Xu M, Shibayama H, Kobayashi H, Yamada K, Ishihara R, Zhao P, Takeuchi T, Yoshida K, Inagaki T, Nokura K. Granulovacuolar degeneration in the hippocampal cortex of aging and demented patients: a quantitative study. Acta Neuropathol 1992; 85: 1-9.

44. Yamazaki Y, Matsubara T, Takahashi T, Kurashige T, Dohi E, Hiji M, Nagano Y, Yamawaki T, Matsumoto M. Granulovacuolar degenerations appear in relation to hippocampal phosphorylated tau accumulation in various neurodegenerative disorders. PLoS One 2011; 6: e26996.
45. Zhu X, Rottkamp CA, Boux H, Takeda A, Perry G, Smith MA. Activation of p38 kinase links tau phosphorylation, oxidative stress, and cell cycle-related events in Alzheimer disease. J Neuropathol Exp Neurol 2000; 59: 880-888. 